\title{
The Dynamics of Proactive and Reactive Cognitive Control Processes in the Human Brain
}

\author{
L. Gregory Appelbaum ${ }^{1}$, C. Nicolas Boehler ${ }^{1,2}$, Lauren A. Davis ${ }^{1}$, \\ Robert J. Won ${ }^{1}$, and Marty G. Woldorff ${ }^{1}$
}

\begin{abstract}
In this study, we leveraged the high temporal resolution of EEG to examine the neural mechanisms underlying the flexible regulation of cognitive control that unfolds over different timescales. We measured behavioral and neural effects of color-word incongruency, as different groups of participants performed three different versions of color-word Stroop tasks in which the relative timing of the color and word features varied from trial to trial. For this purpose, we used a standard Stroop color identification task with equal congruent-to-incongruent proportions (50\%/50\%), along with two versions of the "Reverse Stroop" word identification tasks, for which we manipulated the incongruency proportion (50\%/50\% and 80\%/20\%). Two canonical ERP markers of neural processing of stimulus incongruency, the frontocentral negative polarity incongruency wave $\left(\mathrm{N}_{\mathrm{INC}}\right)$ and the late positive component (LPC), were evoked across the various conditions. Results indicated that color-word
\end{abstract}

\section{INTRODUCTION}

Performance of daily life activities requires flexible and adaptive cognitive control processes that include selection of the most appropriate actions. Such control is particularly necessary when automatic or previously learned behaviors are not optimal for achieving a goal and might interfere with appropriate behavior. Experimental tasks that create such competition or "conflict" between behavioral options have been extensively utilized to study the range of contexts and timescales under which cognitive control processes operate (Mansouri, Tanaka, \& Buckley, 2009; Egner, 2008; Botvinick, Braver, Barch, Carter, \& Cohen, 2001). Such studies indicate that control processes can be divided into two broad classes based on whether they are deployed reactively in response to rapidly changing external conditions or are deployed proactively as a more sustained means to strategically optimize behavior (Funes, Lupianez, \& Humphreys, 2010; Braver, Paxton, Locke, \& Barch, 2009; Braver, Gray, $\&$ Burgess, 2007). This distinction indicates that separable control processes can be characterized based on the temporal dynamics of their engagement, a view that is

${ }^{1}$ Duke University, ${ }^{2}$ Ghent University

(C) 2014 Massachusetts Institute of Technology

the underlying motivation behind the widely held "dual mechanisms of control" framework of cognitive control (Braver, 2012).

Under this temporal framework, reactive adjustments reflect those changes that occur within a trial or from trial to trial to adjust performance based on detection and resolution of recently incurred interference because of conflicting stimulus inputs (Jacoby, Kelley, \& McElree, 1999). This type of reactive process, which has also been referred to as a "late correction" mechanism, generally reflects a more temporally local or microscopic control as it represents changes in response to the very recent history of stimulation (Purmann, Badde, \& Wendt, 2009; Ridderinkhof, 2002). Proactive control, in contrast, results from the anticipation and amelioration of interference before it occurs and therefore generally develops over a longer macroscopic timescale as individuals interact with stimuli in a given context and develop expectations about the likelihood of upcoming conflict. Proactive control is therefore conceptualized as enabling a form of "early selection" in which goal-relevant information is actively maintained in a sustained manner, before the occurrence of cognitively demanding events, to optimally bias cognitive systems in a goal-driven manner (Braver et al., 2007; Miller \& Cohen, 2001). For example, in the 
presence of more frequently occurring conflicting stimuli, individuals tend to increase the amount of top-down control exerted in a task and are therefore less susceptible to conflict from irrelevant distracters. Such "incongruency proportion" manipulations have been widely shown to alter both behavioral (Bugg, Jacoby, \& Chanani, 2010; Crump, Gong, \& Milliken, 2006) and neural (King, Korb, \& Egner, 2012; Tillman \& Wiens, 2011; Egner, 2007; Carter et al., 2000; West \& Alain, 2000) conflict effects. Although considerable progress has been made in mapping out the brain areas that contribute to the cognitive control mechanisms hypothesized in the dual mechanisms of control framework, little is known about the temporal dynamics of the neural processes that underlie these mechanisms.

In previous research, we explored the behavioral and neural underpinnings of proactive and reactive control by measuring behavioral performance and ERPs as participants performed Stroop color identification tasks in which the color and word components were separated in time (Appelbaum, Boehler, Won, Davis, \& Woldorff, 2012; Appelbaum, Meyerhoff, \& Woldorff, 2009). In these studies, Stroop color and word stimuli were separated by SOAs of $-200,-100,0,100$, and $200 \mathrm{msec}$, while ERPs of brain activity were recorded. The high-temporal resolution of the EEG recordings enabled us to study neural processing interactions during Stroop interference to relevant and irrelevant features that occurred separated in time.

In these studies, we focused on two electrophysiological markers of stimulus conflict that have been widely related to stimulus-response incongruency. The first of these, the incongruency negativity $\left(\mathrm{N}_{\text {INC }}\right.$; often referred to as an N450), is a negative polarity ERP wave that is larger in magnitude when evoked by incongruent stimuli as compared with either congruent or neutral stimuli. This component appears from approximately 300 to $550 \mathrm{msec}$ poststimulus over centroparietal scalp locations (Appelbaum et al., 2009, 2012; Tillman \& Wiens, 2011; Larson, Kaufman, \& Perlstein, 2009; West, Jakubek, Wymbs, Perry, \& Moore, 2005; MarkelaLerenc et al., 2004; West, 2003; Liotti, Woldorff, Perez, \& Mayberg, 2000; West \& Alain, 1999) and has been associated with activity in the pFC, specifically ACC (BadzakovaTrajkov, Barnett, Waldie, \& Kirk, 2009; Hanslmayr et al., 2008). This component has been generally related to conflict detection as it is larger for incongruent stimuli, yet can still be evoked after a response has been prepared (Coderre, Conklin, \& van Heuven, 2011).

The second conflict-related component of interest of these previous studies appears as a positive potential deflection over parietal areas and a negative potential deflection over lateral frontal areas, beginning approximately 500 msec after stimulus onset (Appelbaum et al., 2009; Larson et al., 2009; West, 2003; Liotti et al., 2000; West \& Alain, 2000). This component has been referred to by a number of different names, including the late positive component $^{1}$ (LPC; Appelbaum et al., 2012; Donohue,
Liotti, Perez, \& Woldorff, 2012; Coderre et al., 2011; Liotti et al., 2000), the slow positivity (Chen \& Melara, 2009), and the conflict-related slow potential (Larson et al., 2009; West, 2003). This component has been modeled as arising from generators in the middle and/or inferior frontal gyri, as well as from the left extrastriate region (West, 2003), further implicating its role in conflict resolution processes. In addition, the amplitude of this component has been found to correlate with RT and task accuracy, suggesting a role in response selection, rather than in conflict processing, per se (West et al., 2005; Atkinson, Drysdale, \& Fulham, 2003). Alternatively, due in part from the left posterior distribution of this component, other studies have suggested that it may reflect semantic reactivation of the word representation in posterior language areas following signaling of conflict resolution from anterior regions of the brain such as ACC (Liotti et al., 2000). In light of these diverse attributes, the true functional role of this component is still unsettled.

In both of our previous Stroop studies, we found that both behavioral performance effects and the associated neural responses indexed by ERP modulations were highly sensitive to the relative temporal separation between the task-relevant colors and task-irrelevant distracter word information. When color and word components were separated by SOAs that were randomly intermixed from trial to trial (Appelbaum et al., 2009), maximal incongruency effects were observed at the earliest tested preexposure SOAs (i.e., $-200 \mathrm{msec}$ SOA when the word information was presented before the color), with the magnitude of these effects decreasing monotonically with later SOAs. When the SOAs between the relevant color and irrelevant word stimulus components were held constant within each experimental block (Appelbaum et al., 2012), the greatest behavioral and neural incongruency effects occurred for the simultaneous presentation (0 msec SOA). These differences in conflict processing were accompanied by rapid ( $\sim 150 \mathrm{msec})$ modulations of the sensory ERPs to the irrelevant distracter components when they occurred consistently first, suggesting that individuals are able to strategically allocate their attention in time to mitigate the influence of a temporally predictable distracter. Collectively, these findings indicate that individuals may be exploiting different proactive strategies to more effectively filter irrelevant information based on the temporal predictability of SOA trials within an experimental run and that these differences may in turn engender different reactive influences on sensory processing.

Here we significantly expanded upon these findings to investigate neural and behavioral mechanisms underlying the dual mechanisms of control by leveraging both the incongruency proportion manipulation and the widely reported asymmetry in the amount of conflict induced by relatively automatic word reading versus less automatic color naming. For this purpose, we compared the behavioral and neural responses from a standard 
Stroop color identification task with equal congruentto-incongruent proportions (50\%/50\%) and two versions of the "Reverse Stroop" word identification tasks (Durgin, 2000; Stroop, 1935), for which we manipulated the proportion of incongruent trials (50\%/50\% vs. 80\%/20\%). Through these manipulations, we examined how conflict processing is implemented on a macroscopic, contextdependent scale and related $\mathrm{N}_{\text {INC }}$ and LPC activity to behavioral performance to better establish the functional mechanisms underlying cognitive control in the human brain.

\section{METHODS}

\section{Participants}

Seventy-eight normal, neurologically intact participants with normal or corrected-to-normal visual acuity took part in these tasks (ages 18-38 years, 34 women). Thirty-three individuals participated in the Stroop experiment (facets of the data from 20 of these 33 participants have previously been reported elsewhere [Appelbaum et al., 2009]), 30 individuals participated in the Reverse Stroop 50/50 proportion experiment, and 18 individuals participated in the Reverse Stroop 80/20 proportion experiment. Three individuals participated in the Stroop 50/50 task and later $(\sim 1 \mathrm{yr})$ returned to also partake in the Reverse Stroop 50/50 task. (Because these sessions were separated by 10-12 months and because technical as well as psychological aspects can differ between sessions, we analyzed the results from the different experiments in a fully between-group fashion.) All participants were screened with Ishihara plates to confirm normal color vision, and informed consent was obtained before experimentation under a protocol approved by the Duke University Institutional Review Board. Participants were instructed on the task and given practice trials before the start of the experiment. All participants were paid between $\$ 10$ and
Figure 1. SOA design and tasks. (A) In these SOA variants of the Stroop and Reverse Stroop tasks, color-word stimuli were presented with five levels of relative onset timing. As depicted schematically, the target stimulus component (aligned here at $0 \mathrm{msec}$ ), could be preceded by, presented simultaneously with, or followed by the irrelevant stimulus component. Here each of the temporal separations $(-200,-100,0$, +100 , and $+200 \mathrm{msec}$ ) are shown on a separate row indicating the relative timing onsets between the target and distracter elements. Once both stimulus components were presented, they remained on the screen for an additional $1000 \mathrm{msec}$ for all conditions. In the Stroop task (top), the participants' task was to report the physical color of the stimulus while ignoring the meaning of the written word. In the Reverse Stroop task (bottom), the participants were to report the written word while ignoring the physical color. (B) Schematic illustration showing the three tasks. These were composed of two possible "Attentional Goals" (color or word identification) and two relative "Incongruency Proportions" (50\%)

$50 \%$ or $80 \% / 20 \%$,

Congruent/Incongruent).

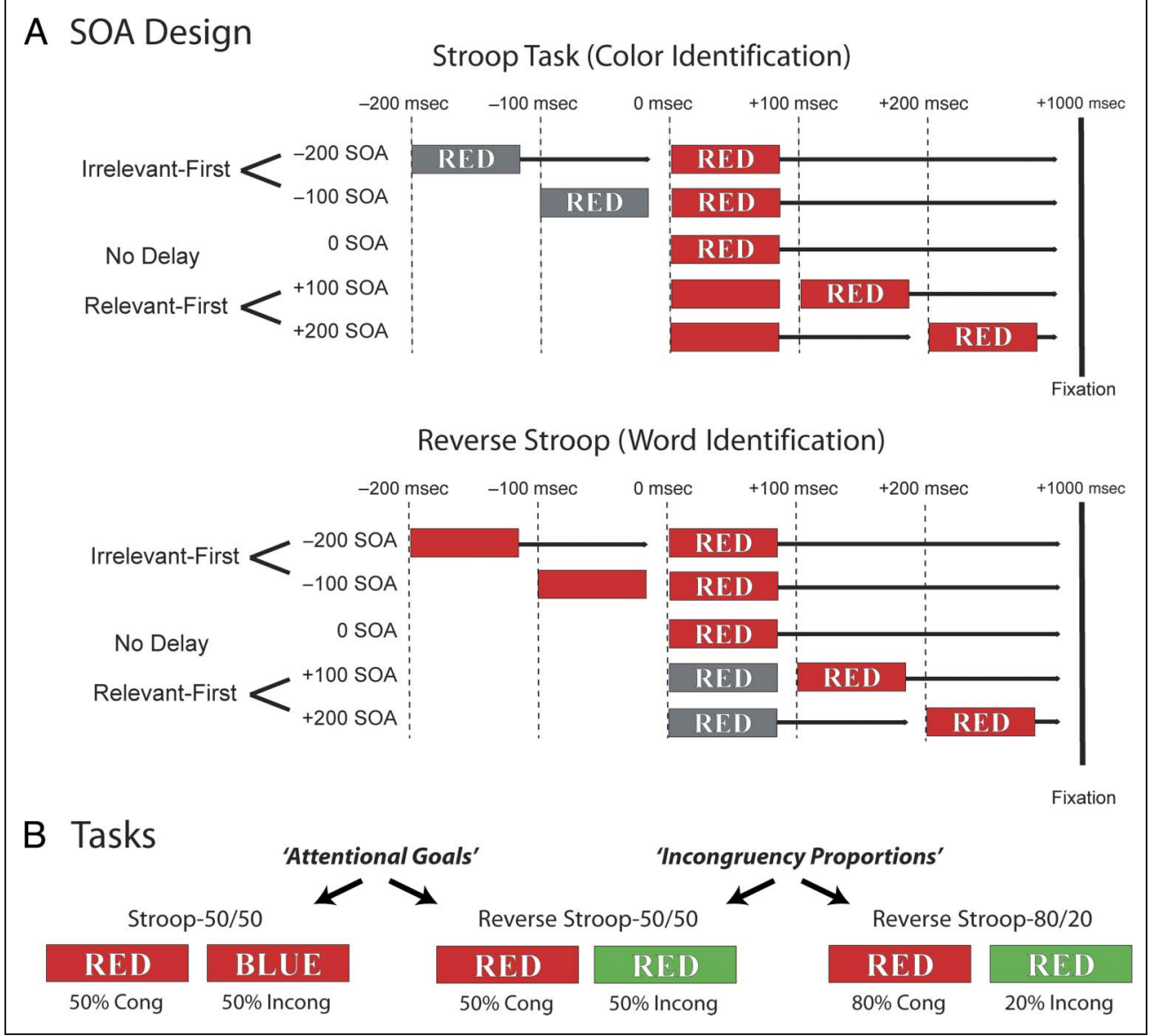


$\$ 15$ per hour for their participation. The data from seven participants were excluded from the final behavioral and ERP analyses because of problematically high levels of EEG artifacts (e.g., eye blinks) or failure to complete the task, leaving 28, 28, and 18 participant sessions in the final analyses for the Stroop 50/50, Reverse Stroop 50/50, and Reverse Stroop 80/20 tasks, respectively.

\section{Experimental Design}

Example experimental stimuli and task parameters are illustrated schematically in Figure 1. Stimuli consisted of red-, green-, blue-, or yellow-colored horizontal rectangles and corresponding English color-word text strings "RED," "GREEN," "BLUE," or "YELLOW," written in white font with black borders and positioned in the center of the colored rectangle. These stimuli were presented on a gray screen (luminance value: $40 \mathrm{~cd} / \mathrm{m}^{2}$ ) with a white fixation cross at the center. Colored rectangles subtended $5^{\circ} \times 16^{\circ}$ and were centered $3.75^{\circ}$ below fixation.

The current experimental design consisted of four independent variables that mapped onto three separate tasks. The first two variables were varied for each participant, whereas the third and fourth variables differed between experimental groups. The first independent variable was Incongruency, which was defined by whether the physical color of the colored bar and the meaning of the written word corresponded or not on each trial. In all experimental sessions, the congruent pairings were split evenly between the corresponding color and word pairs (red-RED, green-GREEN, blue-BLUE, yellow-YELLOW), whereas the incongruent pairings were split evenly between the twelve possible non-corresponding, incongruent pairings (red-GREEN, red-BLUE, red-YELLOW, greenRED, green-BLUE, green-YELLOW, blue-RED, blueGREEN, blue-YELLOW, yellow-RED, yellow-GREEN, yellow-BLUE).

The second independent variable was the SOA between the presentation of the task-irrelevant distracter stimulus component and target stimulus component (shown schematically in Figure 1A). There were five levels of SOA, such that the task-irrelevant stimulus could precede the target stimulus ( -200 and -100 msec conditions), occur simultaneously with it (0 msec or "no-delay") or follow it $(+100$ and $+200 \mathrm{msec})$. For shorthand, these SOAs may be referred to by their relative onset timing: $-200,-100$, $0,+100,+200$, or more generally as negative and positive SOAs (of the task-irrelevant stimulus component relative to the task-relevant one). In all three tasks SOAs were pseudorandomly intermixed across trials and counterbalanced to contain the same numbers of trials in each experimental session at each SOA.

The third independent variable was Attentional Goals (Figure 1B). In separate experimental sessions, different sets of participants were instructed to either perform the Stroop (color identification) task or the Reverse Stroop (word identification) task. In the Stroop task, participants were instructed to report the physical color of the bar as quickly as possible, while ignoring the written word. In the Reverse Stroop task, participants were instructed to report the semantic meaning of the written word as quickly possible, while ignoring the physical color of the bar. In both tasks, responses were given by pressing one of four keys on the keyboard corresponding to four possible targets. For all tasks, the "red" and "green" responses were mapped to the " $\mathrm{D}$ " and " $\mathrm{F}$ " keys of the left hand, and "blue" and "yellow" were mapped to the "J" and "K" keys on the right hand. All four-button mappings were indicated with colored stickers attached to the letter keys (Sugg \& McDonald, 1994).

The fourth independent variable was Incongruency Proportion. In separate experimental sessions, two different combinations of congruent/incongruent trial type probabilities were used. In the equal probability variants, color-bar and color-word combinations matched on half of the trials (congruent, e.g., red-RED), whereas the other half of the trials were split evenly between the possible noncorresponding mappings (incongruent, e.g., red-YELLOW, red-GREEN, and red-BLUE). In the unequal probability variant, congruent stimulus pairings were presented on $80 \%$ of the trials at each of the SOAs, whereas the other $20 \%$ of the trials were split evenly between the possible noncorresponding mappings. In the current experimental design, both the Stroop and Reverse Stroop tasks were collected with equal probabilities (Stroop 50/50 and Reverse Stroop 50/50) to compare the effect of automaticity, control demand, and the nature of the relevant versus irrelevant stimulus dimensions. As this manipulation greatly reduced both the behavioral and neural conflictprocessing effects in the Reverse Stroop task, the incongruency proportion manipulation was applied to examine whether this would restore the conflict-processing effects (Reverse Stroop 80/20).

For all tasks, participants were instructed to maintain central fixation and to minimize eye blinks during the experimental run. RTs and error rates were monitored while 64-channel EEG was recorded. On every trial, the color bar and the color word remained on the screen together for $1000 \mathrm{msec}$ after the onset of the later of the two stimulus components. Individual trials were separated by intertrial intervals that varied randomly between 1300 and $1700 \mathrm{msec}$, during which time only the fixation cross was present on the screen. For most participants $(N=56)$, the experimental sessions consisted of 22 runs of 60 trials each (approximately $1 \mathrm{hr}$ of total experimental stimulation time). Four participants, however, performed 20 runs of 60 trials each, 1 participant performed 18 runs of 60 trials each, and 13 participants performed 28 runs of 48 trials each. These all yielded very similar numbers of effective trials, as indicated by the ranges shown in Table 1 . Before recordings began, participants were given one or two practice runs to learn the mapping of the fourcolor response buttons, and during the experimental 
Table 1. Trial Counts by Condition for the Three Tasks

\begin{tabular}{|c|c|c|c|c|c|c|c|c|c|}
\hline & \multicolumn{3}{|c|}{ Averages } & \multicolumn{3}{|c|}{$S D$} & \multicolumn{3}{|c|}{ Ranges } \\
\hline & Stroop 50/50 & RS 50/50 & RS 80/20 & Stroop 50/50 & RS 50/50 & RS 80/20 & Stroop 50/50 & $R S 50 / 50$ & RS 80/20 \\
\hline$-200 \mathrm{C}$ & 88.7 & 86.4 & 156.3 & 11.4 & 14.6 & 19.2 & 66-111 & $57-113$ & $113-185$ \\
\hline$-200 \mathrm{I}$ & 87.9 & 89.3 & 38.8 & 10.3 & 12.2 & 5.7 & 67-108 & 59-105 & $28-47$ \\
\hline$-100 \mathrm{C}$ & 91.8 & 87.4 & 156.5 & 12.0 & 13.3 & 19.1 & 64-112 & 59-116 & 116-183 \\
\hline$-100 \mathrm{I}$ & 87.6 & 85.3 & 39.9 & 10.7 & 14.5 & 5.2 & 66-109 & 59-115 & $30-46$ \\
\hline $0 \mathrm{C}$ & 92.1 & 87.2 & 158.1 & 11.6 & 13.2 & 20.4 & 69-115 & 58-111 & 113-188 \\
\hline $0 \mathrm{I}$ & 90.5 & 84.8 & 39.7 & 12.4 & 13.3 & 5.6 & $66-115$ & $57-111$ & $28-47$ \\
\hline $100 \mathrm{C}$ & 86.6 & 87.5 & 156.3 & 11.5 & 13.4 & 22.5 & 64-110 & 61-114 & $98-187$ \\
\hline $100 \mathrm{I}$ & 87.5 & 87.0 & 39.8 & 13.1 & 14.5 & 5.7 & 61-114 & 56-118 & $29-48$ \\
\hline $200 \mathrm{C}$ & 91.6 & 87.3 & 153.8 & 11.1 & 13.2 & 25.7 & 69-114 & 59-111 & $107-189$ \\
\hline $200 \mathrm{I}$ & 92.4 & 86.5 & 40.2 & 12.6 & 14.7 & 5.1 & 64-114 & 56-111 & $29-48$ \\
\hline
\end{tabular}

This table lists the average number of trials by condition. The standard deviation of the number of trials per condition and the range of trials per condition (participant-wise minimum and maximum) are listed for each of the three tasks (columns) in each of the 10 conditions SOA by congruencies conditions (rows). Abbreviations: RS = Reverse Stroop; C = Congruent; I = Incongruent.

session participants were given the opportunity to rest between runs.

\section{Data Acquisition and Analysis}

\section{Behavioral Analysis}

Behavioral responses were monitored and recorded as participants performed the tasks and were later analyzed for significant differences. Trials were counted as correct if participants responded correctly between 200 and $1200 \mathrm{msec}$ following the presentation of the target stimulus component. In each condition RT was taken as the time between the onset of the response-relevant feature and button-press response (in msecs). Collectively, all nonregistered trials, which included those in which no buttons were pressed, more than one button press was registered, or button presses fell outside the 200-1200 msec response window, constituted less than $2.5 \%$ of the total trials. As no systematic differences were observed for responses to the different specific target words or colors, data were collapsed over the corresponding color-bar/ color-word combinations to arrive at within-participant mean RTs (correct trials only) and error rates for the congruent and incongruent instances of the five SOA conditions for the different tasks.

An important goal of this study was to establish the relationships between the SOA $\times$ Incongruency interactions within the contexts defined in the three different tasks. For this purpose, mixed-model three-way ANOVA, with within-group factors Incongruency (two levels) and SOA (five levels) and the between-group factor of Tasks (three levels: Stroop 50/50, Reverse Stroop 50/50, Reverse Stroop 80/20), was performed separately on the RT data.
Because the independent variables Attentional Goals and Incongruency Proportion comprise three separate experiments, we use the factor Tasks to describe the between-group comparison that collapses over these two dimensions.

To test for specific differences because of either the Attentional Goals (color vs. word identification in the equal probability tasks) or Incongruency Proportion (50/50 vs. 80/20 in the Reverse Stroop tasks), separate three-way ANOVAs were performed on the individual pairs of tasks. In addition, separate $5 \times 2$ (SOA $\times$ Incongruency) two-way repeated-measures ANOVAs were performed on the RTs to determine significant main effects and interactions of experimental conditions on behavioral performance within each separate task. Specific post hoc comparisons using two-tailed $t$ tests were performed on the congruent versus incongruent RTs, separately for each SOA and each task, to establish the presence of significant behavioral incongruency effects. Alpha levels for these $t$ tests were corrected using Bonferroni corrections for the five levels of SOA in each task (effective significant $p$ value $<.01$ for each test). All ANOVA analyses were corrected where necessary for violations of sphericity using the Greenhouse-Geisser correction. Repeatedmeasures variance was not modeled into the analysis for the three individuals who participated in both of the 50\%/ $50 \%$ tasks, as these represented a small proportion of the total data (74 total sessions).

\section{ERP Recording and Analysis}

The EEG was recorded continuously from 64 channels mounted in a customized, extended-coverage, elastic 
cap (Electro-Cap International, Eaton, $\mathrm{OH}$ ) using a bandpass filter of $0.01-100 \mathrm{~Hz}$ at a sampling rate of $500 \mathrm{~Hz}$ (SynAmps, Neuroscan, El Paco, TX). All channels were referenced to the right mastoid during recording. The positions of all 64 channels were equally spaced across the customized cap and covered the whole head from slightly above the eyebrows to below the inion posteriorly (Woldorff et al., 2002). Impedances of all channels were kept below $5 \mathrm{k} \Omega$, and fixation was monitored with both EOG recordings and a zoom lens camera. Recordings took place in an electrically shielded, sound-attenuated, dimly lit, experimental chamber.

For each participant, ERPs to the onset of the target stimulus component were selectively averaged for each task, SOA, and Incongruency condition. ERP processing included the re-referencing of all channels to the algebraic mean of the two mastoid electrodes. A digital, noncausal, 9-point running average filter was applied to the

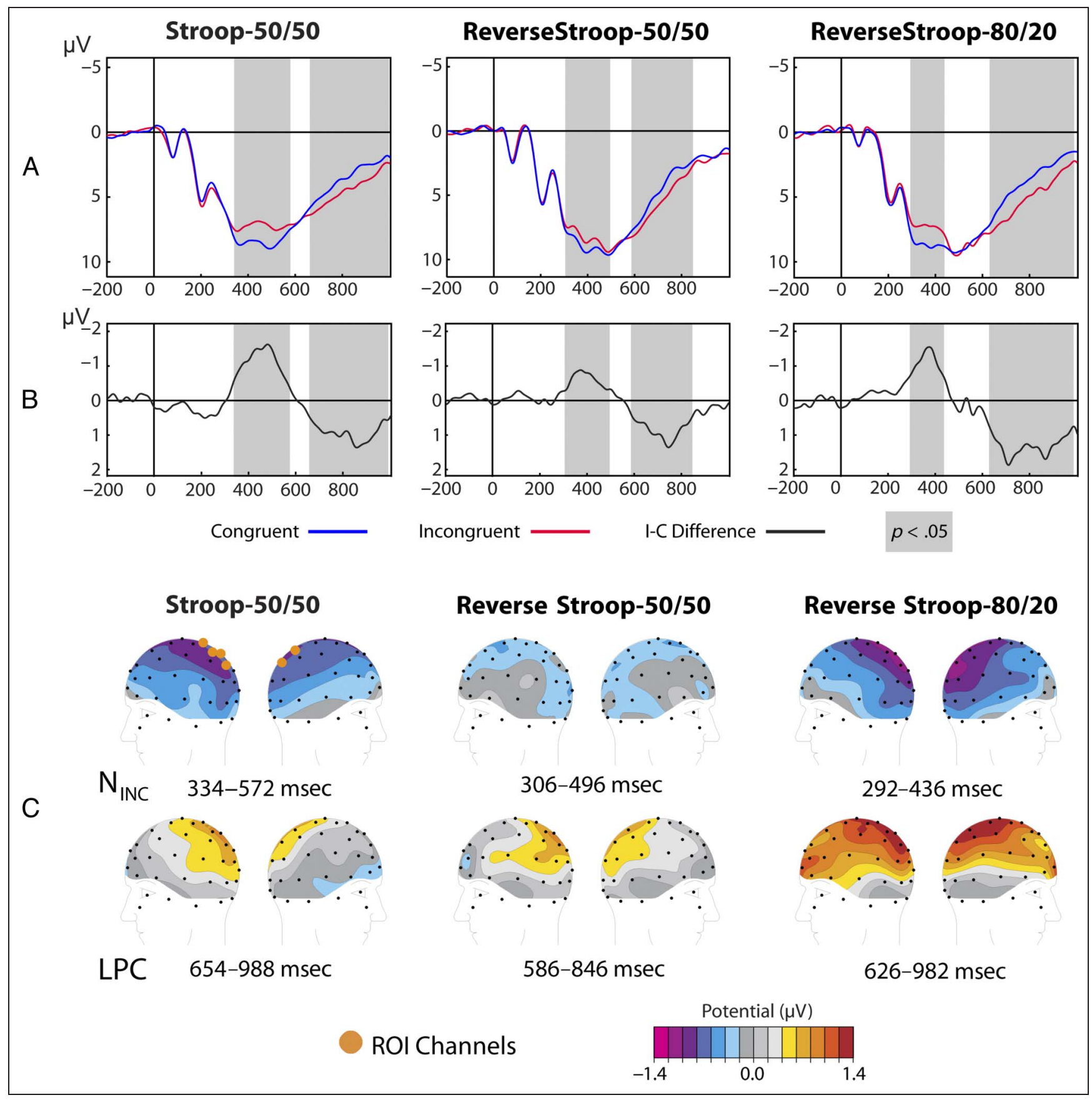

Figure 2. ERP results for the 0-msec SOA conditions. (A) Grand-averaged waveforms computed in the six-channel ROI are shown for incongruent (red) and congruent (blue) trials of the 0-msec SOA condition of the three Attentional Goals. (B) Incongruent minus congruent difference waveforms reveal prominent negative $\left(\mathrm{N}_{\mathrm{INC}}\right)$ and positive (LPC) deflections for each task. (C) Spline-interpolated topographic maps computed over the significant $\mathrm{N}_{\mathrm{INC}}$ and LPC latency ranges (highlighted in gray in A and B and specified below each map in C) are shown for each task. 


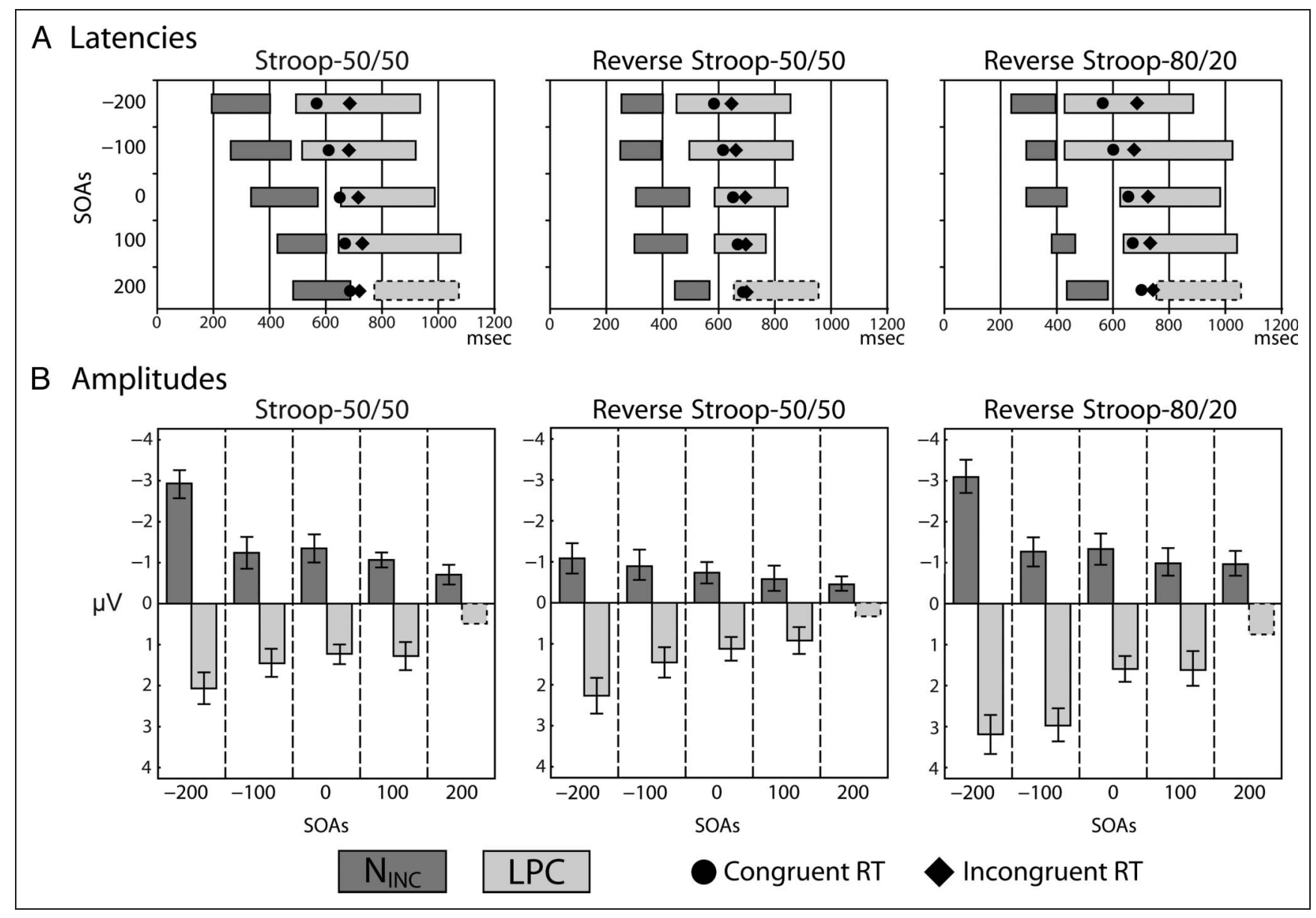

Figure 3. ERP latencies and amplitudes for three Attentional Tasks. (A) Latency ranges of significant within-SOA effects are shown by the dark gray bars for the $\mathrm{N}_{\mathrm{INC}}$ and light gray bars for the LPC for each SOA and task. (B) Mean ERP amplitudes for the $\mathrm{N}_{\text {INC }}$ (negative amplitudes plotted upward) and LPC (positive amplitudes plotted downward) are shown for the five SOAs and three tasks. Hashed outlines for the $+200 \mathrm{msec}$ SOA LPC component indicate that these values were estimated from the local peak window and that these responses did not reach permutation significance. Error bars indicate \pm 1 standard deviation.

ERP averages, which greatly reduces signal at frequencies of $56 \mathrm{~Hz}$ and above at our sampling frequency of $500 \mathrm{~Hz}$ (Talsma \& Woldorff, 2005). Artifact rejection was performed off-line by discarding epochs of the EEG that were contaminated by eye movements or eye blinks (as detected from the EOG recordings), as well as by excessive muscle-related potentials, drifts, or amplifier blocking. The artifact rejection thresholds were preset to $\pm 100 \mu \mathrm{V}$ for vertical eye channels and $\pm 75 \mu \mathrm{V}$ for all others and were applied from -200 to $900 \mathrm{msec}$ around the presentation of the relevant target feature component. These parameters were minimally adjusted for each participant to retain the most trials while eliminating the above sources of contamination and then applied via a computer algorithm that was blind to the specific trial types. These parameters led to an average trial rejection rate of $\sim 16 \%$, which did not differ systematically across tasks or conditions. The average, standard deviation, and range of trials per condition and task are shown in Table 1.
Separate ERPs were computed for correctly reported congruent and incongruent trials (i.e., excluding errors and nonresponses) at each of the five SOAs by timelocking to the onset of the target stimulus. Because no differences were observed in the ERP responses for the different specific target colors, averages were collapsed over all corresponding color-bar/color-word combinations yielding 10 ( 5 SOAs $\times 2$ Incongruency) evoked response types for each of the three tasks. To isolate brain potentials related to color-word compatibility, difference waves were computed separately for each SOA in each task by subtracting the ERPs for congruent trials from the ERPs for incongruent trials. We explicitly focus our ERP analyses on the incongruency difference waves (incongruent trial responses minus congruent trial responses), because the SOA manipulation utilized in these experiments introduces differential amounts of overlap in the ERP averages because of the differential temporal separation between the stimulus components. As this overlap is identical for congruent and incongruent stimuli 
within each SOA condition, the difference wave subtracts off this overlap and while also enabling the isolation of processes related to the Stroop stimulus incongruency, thereby serving as a principled ERP marker for assessing interactions between the SOA and the neural processing related to the conflict processing interactions.

Statistical analysis of the Incongruency, SOA, and Task (comprising both the Attentional Goals and Incongruency Proportion variations) effects were carried out using a six- channel ROI (see orange dots in Figure 3) consisting of posterior-parietal left (P01, P1), right (P02, P2), and midline channels $(\mathrm{CPz}, \mathrm{Pz})$ channels. This ROI was used in previous Stroop ERP studies by our lab (Appelbaum et al., 2012) and closely matches channels reported in other manual Stroop ERP tasks (Donohue et al., 2012; Coderre et al., 2011; Liotti et al., 2000; West \& Alain, 1999).

Using an equally weighted average of these six ROI channels, we first applied permutation testing techniques

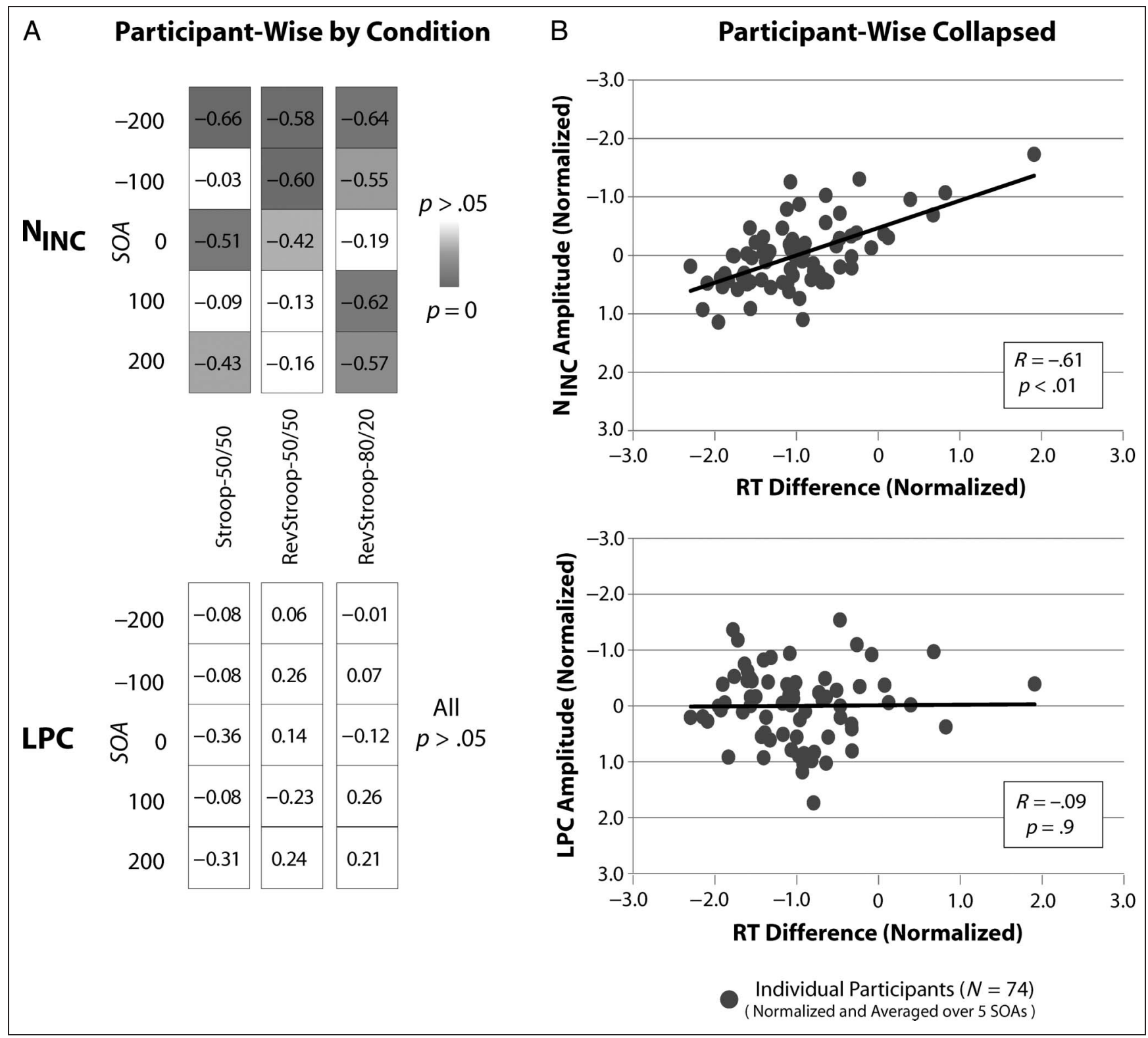

Figure 4. RT versus ERP amplitude relationships are shown through two different types of participant-wise correlations. (A) Participant-wise correlations for each condition are depicted as grayscale maps that relate the normalized RT and normalized $\mathrm{N}_{\text {INC }}$ (top) and LPC (bottom) effect sizes. In these matrices, the numbers depict the Pearson's $R$ coefficients and the grayscale shading indicates the correlational $p$ values across participants within each individual condition. Results indicate that, although the $\mathrm{N}_{\mathrm{INC}}$ amplitude correlated with RT effect sizes across 10 of the 15 experimental conditions (top), LPC amplitudes did not predict RT effect sizes in any of the conditions (bottom). (B) Participant-wise correlations were computed after averaging over all five SOA conditions for each participant, thereby providing a single data point for each individual. Here the $z$ score-normalized RT effect sizes (I-C) are plotted against the $z$ score-normalized $\mathrm{N}_{\mathrm{INC}}$ (top) and LPC (bottom) response magnitudes, averaged over the five SOAs. The linear regression lines, Pearson's $R$ coefficients, and correlational $p$ values indicate there was a significant relationship between the magnitude of the behavioral effect size and the $\mathrm{N}_{\mathrm{INC}}$ component across participants, but that no such relationship existed for the LPC component. 


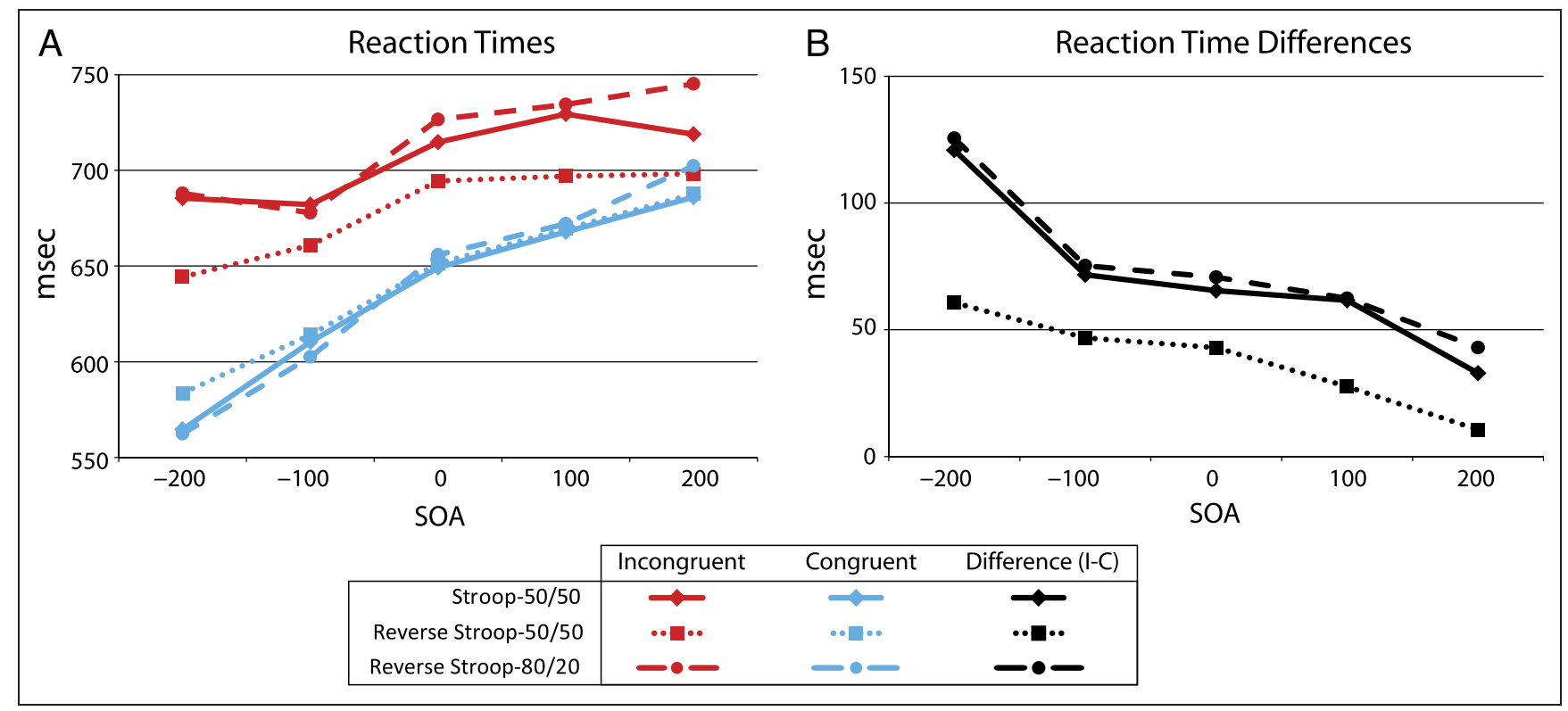

Figure 5. RTs and incongruency RT differences (incongruent minus congruent) for the three tasks. (A) In all three tasks, incongruent trials (red) are slower than congruent (blue) trials. (B) Incongruent minus congruent RT differences (black) are largest at negative SOAs and decline monotonically at later SOAs for all tasks. Overall, the effect sizes across SOAs were greatly reduced (nearly 2 to 1 ) in the Reverse Stroop 50/50 task relative to either of the other two tasks (Stroop 50/50 and Reverse Stroop 80/20), which did not significantly differ from each other.

to determine the latency ranges at which congruent and incongruent waveforms statistically differed from each other. This comparison was made using methods derived by Greenblatt and Pflieger (2004) and provided by the EMSE software package (Source Signal Imaging, San Diego, CA). Under this bootstrapping approach, congruent and incongruent waveforms were derived from the six-channel ROI at each SOA and in each task. Next, the congruent and incongruent condition assignments for each SOA and in each task were randomized across all participants to compute a permutation sample distribution with 10,000 total samples. The observed incongruent minus congruent difference waveform was then compared with the permutation sample distribution to determine latency ranges that produced amplitude values falling in the top $5 \%$ of the distribution sample, corrected for the total number of samples in the waveform (Nichols \& Holmes, 2002). In this manner we were able to determine the latencies at which incongruency effects were present in ERPs uniquely for each condition. In accord with our expectations, this analysis consistently elicited a negative polarity ERP wave associated with incongruency, the $\mathrm{N}_{\text {INC }}$ and the LPC. These latency ranges were then used to identify condition-wise and task-wise amplitude effects as described in the ANOVA analyses below.

To compare the between-group effects of Task and within-group effects of SOA and Incongruency on the ERP, mixed-model three-way ANOVAs using these factors were performed on the difference-wave amplitudes for the $\mathrm{N}_{\mathrm{INC}}$ and LPC. For each participant and at each SOA, the mean amplitude of the difference wave spanning the duration of significant latencies as determined by the within-condition permutation tests were extracted. For the LPC component in the +200 msec SOA, which did not reach within-task significance, mean amplitudes were estimated from a window spanning $\pm 150 \mathrm{msec}$ of the local peak amplitude, which corresponds roughly to the 290 msec average duration of the LPC in the other SOAs (dashed bars in Figure 5). These mean amplitudes were then submitted to a mixed-model ANOVA that included all three tasks, as well as separate pairwise analyses to assess Attentional Goals (color naming vs. word naming) and Incongruency Proportion (50\%/50\% vs. $80 \% / 20 \%$ for the Reverse Stroop data) effects.

To further clarify the relationship between the observed brain activity effects and behavior, two types of ERP behavioral correlations were computed. First, to evaluate the relationship between the pattern of behavioral and ERP effects over SOAs and tasks, Pearson's correlation coefficient were computed between the mean $\mathrm{RT}$ effect and the mean ERP amplitudes in each of the 15 different conditions ( 3 Tasks $\times 5$ SOAs). Next, to evaluate the relationship between behavior and ERP activity across individuals, participant-wise correlations were performed. Here, RT and ERP effect sizes were first $Z$-score normalized within each of the 15 conditions. These normalized effect sizes were then either contrasted directly to derive a correlation coefficient for each condition (see Figure 6A) or were combined across the five SOA conditions to create a single value for each participant (see Figure 6B). All significant correlations were further checked and found to be robust to the removal of outliers. 


\section{RESULTS}

\section{Behavioral Performance}

Robust and statistically significant behavioral effects of stimulus incongruency were observed in all three variants of the task (Figure 2). For all tasks, and at all SOAs, RTs were faster and accuracy was higher for congruent trials (blue) than for incongruent trials (red). Moreover, these incongruency effects interacted with SOA, producing greater overall effect sizes when the irrelevant distracter preceded the target stimulus but also produced significant incongruency effects even when the irrelevant distracter followed the target by 100 or 200 msec. Taskwise differences were also observed, indicating that the composition of trials and behavioral goals of the task interacted with the pattern of SOA-Incongruency effects. In the following sections, we present behavioral results as they were revealed first through between-task omnibus analyses of the RT data to demonstrate the pattern of effects over all experimental variables, then through withintask RT analyses for each of the three tasks separately. Finally, as accuracy was generally very high (95.2\% correct across all conditions) and produced qualitatively similar results to the RT findings, accuracy results are considered only briefly at the end of this section.

\section{Between-task Effects}

For general statistical evaluation of these data, a $2 \times 5 \times 3$ (Incongruency $\times \mathrm{SOA} \times$ Task), mixed-model ANOVA was performed on the RT data. This omnibus ANOVA demonstrated significant main effects of Incongruency, $F(1,71)=$ $324.0, p<.001$, and SOA, $F(2.8,201.7)=329.5, p<.001$, significant two-way interactions between Incongruency and SOA, $F(3.6,252.4)=67.1, p<.001$, Incongruency and Task, $F(2,71)=12.9, p<.001$, and Task and SOA, $F(5.7,201.7)=2.8, p=.015$. These patterns of effects revealed that congruent RTs were faster than incongruent RTs, that earlier (negative) SOAs produced both faster RTs and larger incongruency effects, and that these effects differed according to the task (see below). Additionally, a significant three-way interaction between Incongruency, SOA, and Task was observed, $F(7.1,252.4)=$ $2.9, p=.006$. When collapsing over all other factors, there was no main effect of Task on RTs, $F(1,71)=.20$, $p=.818$.

To investigate the origin of the significant three-way omnibus interaction, separate $2 \times 5 \times 2$ (Congruency $\times$ SOA $\times$ Task) mixed-model ANOVAs were performed on the three possible task pairings (Stroop 50/50 vs. Reverse Stroop 50/50, Stroop 50/50 vs. Reverse Stroop 80/20, and Reverse Stroop 50/50 vs. Reverse Stroop 80/20). Comparisons between each pair of tasks showed that the omnibus interactions described above were driven by differences in the pattern of effects for the Reverse Stroop 50/50 relative to the Stroop 50/50 (two-way, $F(1,54)=31.7, p<.001$; three-way, $F(3.4,183.9)=4.8, p=.002)$ and relative to the Reverse Stroop 80/20 (two-way, $F(1,44)=17.7, p<$ .001 ; three-way, $F(3.5,155.9)=5.1, p=.001)$. However, no differences were found in any two-way or three-way interactions for the Stroop 50/50 vs. Reverse Stroop 80/20 tasks (two-way, $F(1,44)=0.2, p=.641$; three-way, $F(3.4$, $151.2)=0.13, p=.96)$. It can therefore be concluded that when the congruent and incongruent trial types were presented in equal proportions, RT effects were reduced by nearly half in the word identification task, relative to the color identification task. These effects, however, were essentially completely recovered by reducing the frequency of incongruent trials so that they occurred on only $20 \%$ of the trials, relative to $50 \%$ of the trials.

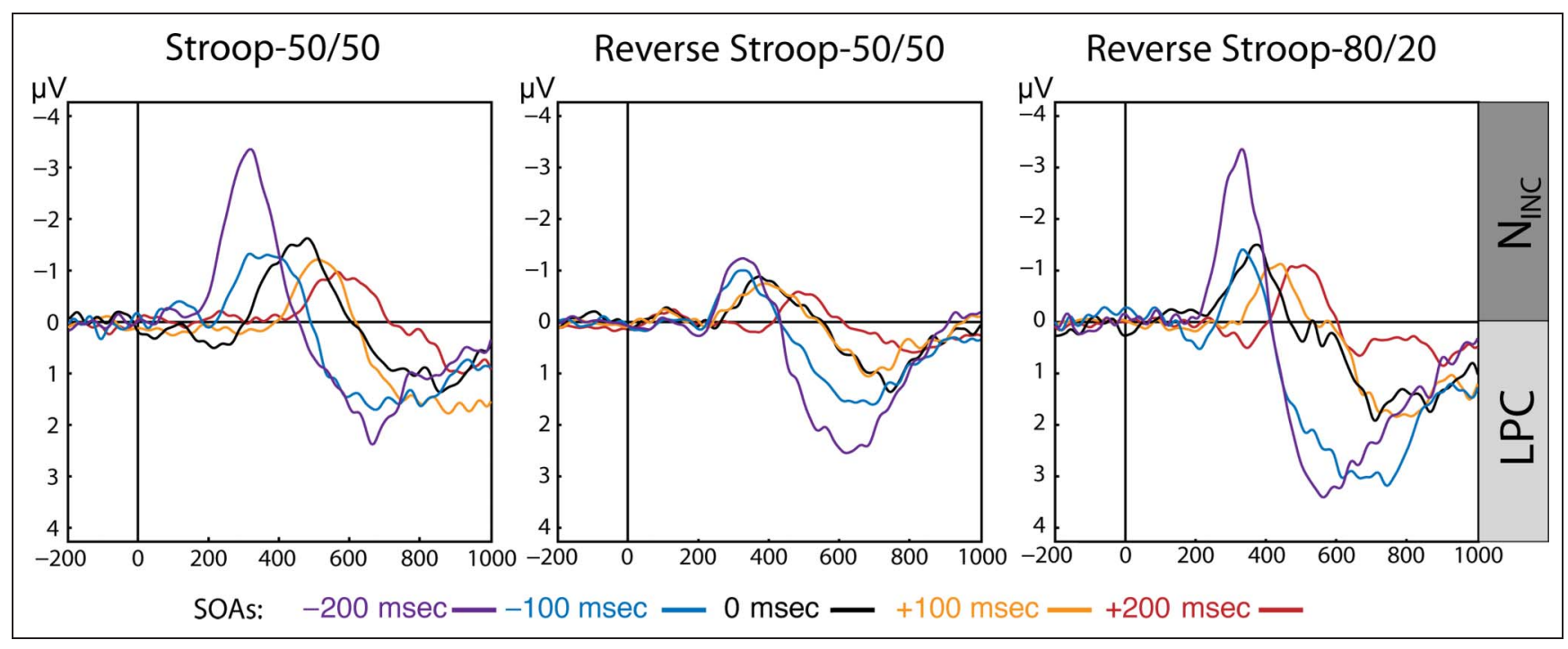

Figure 6. Incongruency difference waves averaged over the six-channel ROI are shown for all SOAs in the three tasks. All waveforms are shown time-locked to the onset of the relevant target component of the stimulus for the different tasks. $\mathrm{N}_{\mathrm{INC}}$ and LPC effects are indicated by the dark and light gray shading, respectively. 
Table 2. Within-task (A) ANOVA and (B) Paired Comparison Results

\begin{tabular}{|c|c|c|c|c|c|c|c|c|c|}
\hline \multirow[b]{2}{*}{ ANOVA } & \multicolumn{3}{|c|}{ Stroop 50/50 } & \multicolumn{3}{|c|}{ Reverse Stroop 50/50 } & \multicolumn{3}{|c|}{ Reverse Stroop 80/20 } \\
\hline & \multicolumn{2}{|r|}{$F(d f)$} & Sig. & \multicolumn{2}{|r|}{$F(d f)$} & Sig. & \multicolumn{2}{|r|}{$F(d f)$} & Sig. \\
\hline Cong & \multicolumn{2}{|c|}{$F(1,27)=191.4$} & $p<.001$ & \multicolumn{2}{|c|}{$F(1,27)=180.3$} & $p<.001$ & \multicolumn{2}{|c|}{$F(1,17)=53.4$} & $p<.001$ \\
\hline SOA & \multicolumn{2}{|c|}{$F(2.5,66.8)=80.9$} & $p<.001$ & \multicolumn{2}{|c|}{$F(2.8,77.1)=143.8$} & $p<.001$ & \multicolumn{2}{|c|}{$F(2.8,47.5)=151.4$} & $p<.001$ \\
\hline Cong $\times$ SOA & \multicolumn{2}{|c|}{$F(3.1,84.9)=24.5$} & $p<.001$ & \multicolumn{2}{|c|}{$F(3.7,99)=28.8$} & $p<.001$ & \multicolumn{2}{|c|}{$F(3.2,54.8)=22.3$} & $p<.001$ \\
\hline \multicolumn{4}{|l|}{$B$} & & & & & & \\
\hline$t$ Tests & $\Delta R T$ & $\mathrm{t}$ & Sig. & $\Delta R T$ & $\mathrm{t}$ & Sig. & $\Delta R T$ & $\mathrm{t}$ & Sig. \\
\hline$-200 \mathrm{msec}$ & 120.8 & $t(27)=13.8$ & $p<.001$ & 60.7 & $t(27)=10.8$ & $p<.001$ & 125.6 & $t(17)=11.3$ & $p<.001$ \\
\hline$-100 \mathrm{msec}$ & 71.7 & $t(27)=11.0$ & $p<.001$ & 46.8 & $t(27)=14.5$ & $p<.001$ & 75.3 & $t(17)=8.0$ & $p<.001$ \\
\hline $0 \mathrm{msec}$ & 65.5 & $t(27)=8.6$ & $p<.001$ & 42.9 & $t(27)=10.4$ & $p<.001$ & 70.7 & $t(17)=6.3$ & $p<.001$ \\
\hline $100 \mathrm{msec}$ & 61.6 & $t(27)=9.1$ & $p<.001$ & 27.6 & $t(27)=7.1$ & $p<.001$ & 62.3 & $t(17)=4.5$ & $p<.001$ \\
\hline $200 \mathrm{msec}$ & 32.9 & $t(27)=3.9$ & $p=.001$ & 10.6 & $t(27)=2.5$ & $p=.015$ & 43 & $t(17)=3.3$ & $p=.004$ \\
\hline
\end{tabular}

Significant main effects and interactions were present for all three tasks. Similarly, significant incongruency effects were present for all SOAs in all tasks (note that two-tailed $t$ test significances are reported). Sig. $=$ significance.

\section{Within-task RT Effects}

To evaluate the pattern of RT effects for each of the three tasks, separate $2 \times 5$ (Congruency $\times$ SOA) ANOVAs were performed (Table $2 \mathrm{~A})$. These analyses revealed significant main effects of Incongruency and SOA, as well as significant Incongruency $\times$ SOA interactions for all three experiments. Post hoc paired comparisons between the congruent and incongruent RTs for each SOA, in each of the three tasks (Table 2B), demonstrated that significant incongruency effects were present in all of the experimental conditions except for the +200 msec SOA condition for the Reverse Stroop 50/50 task (Bonforroni-corrected for five SOA comparisons, $p<.01$ ). Mean RTs and standard errors are presented for each task and condition in Table 3A.

\section{Behavioral Accuracy Effects}

Overall accuracy was high across all conditions and generally mirrored the RT effects reported above. A three-way omnibus ANOVA performed on the error rates revealed significant main effects of Incongruency, $F(1,71)=$ 53.0, $p<.001$, and SOA, $F(3.6,257.4)=4.99, p=.001$, and a significant two-way interaction between Incongruency and SOA, $F(3.5,250.6)=7.65, p<.001$. Although these results should be considered in the context of potential ceiling effects, these patterns of effects indicate that accuracy was higher on congruent trials $(M=96.2)$ than incongruent trials $(M=94.3)$ and that earlier (negative) SOAs produced both higher error rates and larger incon- gruency effects. When collapsing over all other factors, there was no main effect of Task on accuracy, $F(1,71)=$ $.63, p=.535$. Mean accuracy and standard errors are presented for each task and condition in Table 3B.

\section{ERPs}

As in our previous Stroop SOA studies (Appelbaum et al., 2009, 2012), analyses of the data in this study focused primarily on the incongruency difference waves obtained by subtracting the ERPs for congruent trials from those of incongruent trials at each SOA. We explicitly focus our ERP analyses on these incongruency difference waves, because the SOA manipulation utilized in these experiments introduces differential amounts of overlap in the ERP record depending on the temporal separation between stimulus components. As this overlap is equivalent for the congruent and incongruent stimuli within each SOA condition, the difference wave subtracts out this overlap thereby isolating processes related to the Stroop stimulus incongruency effects at each SOA. Accordingly, it serves as a principled ERP marker for assessing interactions between the SOA and the neural processing related to the conflict processing interactions.

In the following section we present first the incongruency subtraction for the 0-msec SOA to illustrate the canonical Stroop $\mathrm{N}_{\text {INC }}$ and LPC incongruency-related ERP components. This is then followed by qualitative depiction and quantitative assessments of the Incongruency $x$ SOA and Incongruency $\times$ SOA $\times$ Task interactions, as 
revealed through their influence on the amplitudes and latencies of the $\mathrm{N}_{\mathrm{INC}}$ and LPC components.

\section{Incongruency Effects at the 0-msec SOA}

Figure 3 illustrates the incongruency subtraction for each of the three tasks in the 0-msec SOA condition. In all three variants, ERP waveforms for congruent (blue) and incongruent (red) color-word pairs diverge roughly 300 msec following the presentation of the target stimulus feature (Figure 3A). As observed in previous studies (Hanslmayr et al., 2008; West, 2003; Liotti et al., 2000; West \& Alain, 1999) the grand-averaged difference waves of the incongruent minus congruent subtraction (Figure 3B) contain relatively early-latency ( 300-500 msec) negative deflections (the $\mathrm{N}_{\mathrm{INC}}$ ) and longer-latency $(\sim 600$ $900 \mathrm{msec}$ ) positive deflections (the LPC). For each task, the $\mathrm{N}_{\text {INC }}$ and LPC components reached statistical significance for protracted latency ranges according to permutation tests comparing the incongruent versus congruent waveforms over the six-channel ROI (indicated by the orange dots). These significant latency ranges are depicted by the gray-shaded bars overlaid on the waveforms and are listed in Table 4.

The spatial distribution of the $\mathrm{N}_{\mathrm{INC}}$ and LPC components for the 0 -msec SOA condition can be seen in Figure 3C. These maps depict the topographic voltage distributions averaged over the full duration for which each component reached statistical significance. For each task, the $\mathrm{N}_{\text {INC }}$ component shared similar medial-central topographies that overlapped with the six-channel ROI but was slightly more posterior in the Reverse Stroop 80/20 case. The LPC component occurred at longer latencies and was consistently maximal over medial centralparietal electrode sites.

\section{Incongruency Effects as a Function of SOA, Attentional Goals, and Incongruency Proportion}

Varying the temporal separation of the color and word components of these stimuli modulated the timing of arrival of conflicting stimulus input to the brain and thus to the areas that detect and resolve conflict. To illustrate how SOA interacts with Incongruency in the three tasks,

Table 3. RTs and SEs (in Parentheses) for Each Condition, SOA, and Task

\begin{tabular}{|c|c|c|c|c|c|}
\hline & -200 & -100 & 0 & 100 & 200 \\
\hline \multicolumn{6}{|l|}{ A. RTS } \\
\hline \multicolumn{6}{|l|}{ Stroop } \\
\hline Cong & $564.6(17.7)$ & $610.3(17.2)$ & $649.2(17.2)$ & $667.8(17.5)$ & $686.0(18.8)$ \\
\hline Incong & $685.4(17.2)$ & 682.0 & $714.7(20.5)$ & $729.4(21.2)$ & 718.9 (23.2) \\
\hline \multicolumn{6}{|l|}{ RS 50/50 } \\
\hline Cong & $583.6(18.3)$ & $614.1(17.7)$ & $651.4(16.5)$ & $669.4(16.8)$ & $687.6(17.3)$ \\
\hline Incong & $644.3(15.6)$ & $660.9(16.7)$ & $694.4(15.8)$ & $696.9(17.4)$ & $698.2(17.3)$ \\
\hline \multicolumn{6}{|l|}{ RS 80/20 } \\
\hline Cong & $562.3(19.5)$ & $602.5(18.5)$ & $655.8(18.7)$ & $672.0(18.5)$ & $702.3(18.8)$ \\
\hline Incong & $687.9(22.0)$ & 677.7 (19.6) & $726.5(23.7)$ & 734.4 (23.9) & $745.2(27.0)$ \\
\hline \multicolumn{6}{|c|}{ B. Accuracy } \\
\hline \multicolumn{6}{|l|}{ Stroop } \\
\hline Cong & $96.4(0.7)$ & $96.1(0.7)$ & $95.9(0.8)$ & $95.3(0.8)$ & $95.1(1.1)$ \\
\hline Incong & $92.8(1.1)$ & $93.7(0.8)$ & $93.4(1.0)$ & $93.6(1.1)$ & $94.9(0.9)$ \\
\hline \multicolumn{6}{|l|}{ RS 50/50 } \\
\hline Cong & $96.5(0.6)$ & $95.4(0.6)$ & $96.1(0.6)$ & $95.9(0.5)$ & $96.2(0.7)$ \\
\hline Incong & $94.1(0.8)$ & $93.9(0.7)$ & $94.4(0.8)$ & $95.6(0.7)$ & $95.7(0.7)$ \\
\hline \multicolumn{6}{|l|}{ RS 80/20 } \\
\hline Cong & $97.0(0.5)$ & $97.1(0.6)$ & $96.7(0.5)$ & $97.7(0.5)$ & $97.5(0.4)$ \\
\hline Incong & $92.4(1.7)$ & $93.9(1.4)$ & $94.0(1.2)$ & $95.7(0.9)$ & $96.5(0.8)$ \\
\hline
\end{tabular}

Cong $=$ congruent Incong $=$ incongruent; RS $=$ Reverse Stroop 
Table 4. Summary of Significant $\mathrm{N}_{\text {INC }}$ and LPC Latencies for the Three Task Variants

\begin{tabular}{lccc}
\hline & Stroop 50/50 & $\begin{array}{c}\text { Reverse } \\
\text { Stroop 50/50 }\end{array}$ & $\begin{array}{c}\text { Reverse } \\
\text { Stroop 80/20 }\end{array}$ \\
\hline Latency & Ranges of Significant & $N_{\text {INC Component }}$ Activity \\
-200 & $194-402$ & $254-402$ & $238-396$ \\
-100 & $262-476$ & $250-396$ & $292-396$ \\
0 & $334-572$ & $306-496$ & $292-436$ \\
+100 & $428-602$ & $300-488$ & $382-466$ \\
+200 & $484-688$ & $444-568$ & $436-582$ \\
Latency & Ranges of Significant & & \\
-200 & $494-936$ & $450-856$ & $428-886$ \\
-100 & $516-920$ & $496-864$ & $428-1026$ \\
0 & $654-988$ & $586-846$ & $626-982$ \\
+100 & $646-1080$ & $586-768$ & $638-1042$ \\
+200 & $777-1077^{\#}$ & $662-962^{\#}$ & $760-1060^{\#}$ \\
\hline
\end{tabular}

Latency ranges of significant $(p<.05)$ run-length-corrected permutation comparisons between incongruent and congruent waveforms for each SOA are indicated for the three tasks. The "\#" indicates that these latencies are estimated from local peak latency, because their activity levels did not reach significance in the permutation tests.

incongruent-minus-congruent difference waves are shown for the six-channel ROI for each SOA condition in Figure 4. Two primary observations are easily visible in these waveforms. First, for each task the amplitudes and latencies of the $\mathrm{N}_{\text {INC }}$ and LPC component followed a roughly monotonic pattern, with later SOAs corresponding to reduced amplitudes and longer latency effects. Second, the overall amplitude of the $\mathrm{N}_{\mathrm{INC}}$ and LPC components appear to vary over the three different tasks.

To quantitatively assess the latency and amplitude profiles of these components, we took a two-step analytical approach. First, as with the 0-msec SOA described above, permutation tests comparing the incongruent versus congruent waveforms were performed to determine significant latency ranges at which the $\mathrm{N}_{\text {INC }}$ and LPC components were evoked for each SOA and task. Next, the between-task and within-task effects were evaluated by comparing the mean response amplitudes for the incongruency effect within these significant latency ranges. As with the behavioral analyses, these ERP effects were evaluated first by assessing the three-way interactions over all three tasks, then separately for the individual pairs of tasks, and finally within each task.

As determined by permutation tests, significant $\mathrm{N}_{\mathrm{INC}}$ activity was evoked at all SOAs in all three tasks. Significant LPC activity appeared at all SOAs except for the +200 SOA, where it did not reach statistical criterion in any of the tasks. The latencies at which these effects reached statistical significance are listed in Table 4 and depicted graphically by the horizontal gray bars in Figure $5 \mathrm{~A}$. For both the $\mathrm{N}_{\text {INC }}$ and LPC, the difference wave activity showed a largely monotonic shift in latency across the SOA conditions. $\mathrm{N}_{\text {INC }}$ activity was generally more transient, lasting an average of $125 \mathrm{msec}$, whereas LPC activity was more sustained and reached criterion significance across a $290-\mathrm{msec}$ window on average (except for at the $+200 \mathrm{msec}$ SOA, as mentioned above). Furthermore, whereas $\mathrm{N}_{\text {INC }}$ activity preceded the RTs for both congruent (circles) and incongruent (diamonds) trial types in all cases, LPC activity generally overlapped with the time range when participants responded behaviorally.

Mean amplitudes derived for the $\mathrm{N}_{\text {INC }}$ component (dark gray bars) and LPC (light gray bars) are shown in Figure 5B for each of the SOAs for each of the tasks. The largest ERP incongruency effects were seen for the negative SOAs, decreasing monotonically over the later SOAs. Thus, the ERP effects paralleled the behavioral results for these tasks (Figure 2), which indicated a clear pattern of SOA-related priming in which the pretarget presentation of the interfering stimulus dimension resulted in greater RT incongruency effects.

ERPs computed with vastly different numbers of trials are potentially susceptible to biases in the relative signal to noise in the averaged waveforms (Luck, 2005). In the Reverse Stroop 80/20 condition, congruency comparisons between the more frequent congruent and less frequent incongruent trials (see Table 1 for trial counts) represent such a case. Therefore, supplementary analyses were conducted to evaluate the influence of trial counts in these data. For this purpose, congruent ERPs were regenerated from a randomly resampled distribution of trials so that they matched the number of incongruent trials at the same SOA for each condition. Paired comparisons on the mean voltage in each of the effect windows (see Table 3) revealed no differences between the original and resampled waveforms at any of the relevant latencies (all $p>3$ ). We therefore infer that the present effects and effect differences between tasks are not driven by relative number of trials included in the ERP.

$N_{I N C}$. Mixed-model ANOVA conducted on the mean $\mathrm{N}_{\text {INC }}$ amplitudes of the three tasks confirmed a withinparticipant main effect of SOA, $F(3.5,20.3)=6.5, p<$ .001 , where earlier SOAs produced larger amplitude responses. In addition, there was also a significant betweenparticipant main effect of Task, $F(2,71)=9.0, p<.001$, as well as an SOA $\times$ Task interaction, $F(7.1,252.3)=2.6, p=$ .013 , indicating differing patterns of incongruency for the different tasks. Separate ANOVAs performed as pairwise comparisons from among the three tasks revealed that both the main effect of Task and the SOA $\times$ Task interaction were primarily driven by a reduction in the $\mathrm{N}_{\mathrm{INC}}$ amplitude for the Reverse Stroop 50/50 task relative to the other two tasks (Reverse Stroop 50/50 vs. Stroop 50/50: interaction, $F(3.4,183)=3.7, p=.01$, main effect, $F(1$, $54)=14.5, p<.001$; Reverse Stroop 50/50 vs. Reverse 
Stroop 80/20: interaction, $F(3.3,147.6)=4.3, p=.04$; main effect, $F(1,44)=14.4, p<.001)$. No difference, however, was present between the Stroop 50/50 task and the Reverse Stroop 80/20 task (interaction, $F(3.6,158.9)=$ $0.11, p=.97$; main effect, $F(1,44)=0.16, p=.68)$. These contrasts therefore indicate that the $\mathrm{N}_{\text {INC }}$ component was sensitive to both the Attentional Goals and Incongruency Proportion task manipulations, as well as to the SOA between stimulus components.

LPC. Mixed-model ANOVA conducted on the mean LPC amplitudes of the three tasks (including the $+200 \mathrm{msec}$ SOA amplitudes estimated from the $\pm 150 \mathrm{msec}$ surrounding the local peak latency) revealed a significant main effect of SOA, $F(3.6,259.77)=19.9, p<.001$, indicating that, like the $\mathrm{N}_{\text {INC }}$ component, LPC amplitudes were larger with earlier SOAs. This ANOVA also a showed significant main effect of Task, $F(2,71)=3.5, p=.03$, although the Task by SOA interaction did not reach statistical significance, $F(7.3,259.7)=1.0, p=.403$. Separate ANOVAs between pairs of the three tasks revealed that the main effect of Task was primarily driven by greater LPC amplitudes for the Reverse Stroop 80/20 task than the other two (vs. Stroop 50/50, F(1, 44) = $4.3, p=.045$; vs. Reverse Stroop 50/50, $F(1,44)=6.7$, $p=.013)$. Thus, the LPC component was sensitive to the Incongruency Proportion manipulation, but not to the attentional instructions defined by the Attentional Goals.

\section{Correlations between ERP Incongruency Effect Amplitudes and Incongruency-related RT Slowing}

The results presented thus far suggest possible functional differences between the $\mathrm{N}_{\mathrm{INC}}$ and LPC components. To further assess these differences and the relationship between these ERP effects and behavior, we computed Pearson's correlation coefficients between the incongruent minus congruent RT differences and the amplitudes of the $\mathrm{N}_{\text {INC }}$ and LPC incongruency ERP effects. These comparisons were done in three ways. First, to evaluate the relationship between the pattern of behavioral and ERP effects over the experimental factors (SOA and task), correlations were computed between the group-mean RT effect and the group-mean ERP amplitudes in each of the 15 different conditions (i.e., averaged over participants for each combination of the three tasks and five SOAs). These comparisons reveal significant positive correlations between the magnitude of the $\mathrm{N}_{\text {INC }}$ and the magnitude of the incongruencyrelated RT slowing effect $(R=-0.95 ; p<.01)$, as well as between the magnitude of the LPC and RT effects $(R=0.81 ; p<.01)$. These relationships can be seen graphically by comparing the pattern of behavioral effects in Figure 2B with the patterns of ERP amplitude effects in Figure 5B. This observation that both of the ERP components correlated with the RT effect sizes across condi- tions indicates that these two ERP components are tracking the overall level of incongruency introduced by the Task and SOA manipulations introduced in these experimental designs.

Next, to evaluate if increased behavioral incongruency effects correlated with larger ERP effects on an individualby-individual basis, participant-wise correlations were performed in two ways. Figure 6 A shows two $3 \times 5$ matrices conveying the participant-wise correlations for each of the 15 experimental conditions for the $\mathrm{N}_{\text {INC }}$ (top) and LPC (bottom). As indicated by the grayscale shading, 10 of the 15 cells for the $\mathrm{N}_{\text {INC }}$ component are significantly (negatively) correlated, indicating that in most of the conditions individuals who showed larger RT effects also produced larger $\mathrm{N}_{\mathrm{INC}}$ amplitudes. In contrast, participant-wise correlations were not significant for any of the 15 cells for the LPC (all $p>.05$ ), revealing no systematic relationship between within-trial RT effects sizes and LPC amplitudes. To assess the overall population relationship, participant-wise correlations were computed collapsed over all five SOA conditions for each participant, thereby providing a single data point for each individual. These population relationships, shown in Figure $6 \mathrm{~B}$, reveal a clear pattern wherein the magnitude of the $\mathrm{N}_{\mathrm{INC}}$ effect correlated with the amount of incongruency-related RT slowing across the participants $(R=$ $-0.61 ; p<.01)$, whereas no such relationship was found between the LPC component and the RT effects $(R=$ $-0.09 ; p=.9)$.

\section{DISCUSSION}

In this study, we exploited the high temporal resolution of EEG to investigate processing interactions in the human brain using the three variants of the Stroop and Reverse Stroop tasks in which the task-relevant and taskirrelevant features were presented with varying temporal separations and with different proportions of congruency trial types. We observed that behavioral and neural incongruency effects were present for all tasks and occurred in all SOA conditions tested. ERP difference waves in all three tasks produced two canonical components associated with stimulus incongruency, the earlier-latency negative wave $\left(\mathrm{N}_{\text {INC }}\right)$ and the longer-latency LPC, that have previously been reported in the literature (Coderre et al., 2011; Bruchmann, Herper, Konrad, Pantev, \& Huster, 2010; Badzakova-Trajkov et al., 2009; Larson et al., 2009; Hanslmayr et al., 2008; Atkinson et al., 2003; West, 2003; Liotti et al., 2000; West \& Alain, 1999). As observed for other manual variants of the Stroop tasks, the $\mathrm{N}_{\text {INC }}$ here appeared as a centrally distributed negative deflection peaking at around $450 \mathrm{msec}$ following the initial stimulus exposure, wheras the LPC appeared as a more sustained and later parietal positive wave. These electrophysiological components were sensitive to somewhat different aspects of the experimental design factors, suggesting 
dissociable functional relationships between the underlying brain mechanisms and behavior. In the following two sections, we discuss how these patterns of findings map onto reactive and proactive accounts of cognitive control, as well as where further research is needed to more fully disentangle the mechanistic interpretation of these effects.

\section{Reactive Control on a Microscopic Timescale}

Humans possess dynamic and flexible cognitive control mechanisms that can be deployed rapidly to address situations in which environmental stimuli conflict with internal goals. This form of reactive control has been proposed to operate as a "late attentional correction" that is mobilized as needed (Jacoby et al., 1999) and has been studied through a host of experimental techniques that assess how performance is adjusted within a trial (e.g., activation suppression) or from trial to trial (e.g., conflict adaptation, negative priming) following the occurrence of conflicting sensory signals. In fact, numerous fMRI (reviewed in Egner, 2007) and ERP (Donohue et al., 2012; Bailey, West, \& Anderson, 2010; Larson et al., 2009; Sturmer, Leuthold, Soetens, Schroter, \& Sommer, 2002) studies have harnessed the congruency sequence effect to tease apart brain regions hypothesized to participate in different control operations (reviewed in Carter \& van Veen, 2007).

In the present design, we utilized another type of microlevel stimulus manipulation that allowed us to assess how conflict-related control mechanisms are deployed over short time frames, depending on the temporal composition of the stimuli. Specifically, relevant and irrelevant colors and words were presented with temporal separations ranging from -200 to $+200 \mathrm{msec}$. This SOA manipulation allows us to determine how reactive control is deployed to address differing level of conflict created by different temporal arrangements. In all three tasks, we observed the greatest behavioral and electrophysiological effects when the irrelevant stimulus component preceded the task-relevant target, with these effects falling off in amplitude at later occurrences of the distracter element. We interpret this pattern as reflecting a form of "conflict-related priming." Here, the earlier presentation of an irrelevant stimulus gives the brain a "head start" in the processing of that stimulus, thereby priming the associated response selection and resulting in a greater competitive advantage when the colors and words match and an increase in interference when they do not. When the target is followed in time by an irrelevant distracter, incongruency effects are reduced in amplitude and occur later in time, reflecting a diminishing influence of the distracter.

This influence can be explained in the context of classic models of forced-choice decision-making (e.g., Ratcliff, 1978) in which evidence for a decision accumulates over time until a response threshold is reached.
When the task-relevant stimulus component is presented first, processing proceeds unimpeded, allowing more evidence to accumulate in favor of the appropriate response before the introduction of the irrelevant stimulus. Under these circumstances, the processing of the irrelevant stimulus has to catch up to that of the relevant one to have any effect, therefore leading to smaller behavioral interference effects and small and later neural effects when presented after a temporal delay. Collectively, these patterns of SOA effects are supported by results from Coderre and colleagues (2011), who also observed conflict-related priming in a Stroop task with SOA separations of -400 , 0 , and $+400 \mathrm{msec}$, as well as from similar cross-modal Stroop tasks from our group (Donohue, Appelbaum, Park, Roberts, \& Woldorff, 2013).

Furthermore, although the present SOA design does not lend itself well to sequential trial analyses (due what would be a complicated combination of sequences and SOAs), other ERP studies have begun to explore for the presence of reactive control as it is expressed through sequential trial conflict adaptation on the $\mathrm{N}_{\text {INC }}$ and LPC components. For example, in recent experiments using visual (Larson et al., 2009) and auditory (Donohue et al., 2012) Stroop tasks, it has been observed that although the LPC differentiated current trial compatibility on the basis of previous trial context, no such conflict adaptation effects were observed with the $\mathrm{N}_{\text {INC }}$. As noted by Larson and colleagues, these finding are somewhat surprising given that $\mathrm{N}_{\mathrm{INC}}$ has been typically modeled as arising from generators in the dorsal ACC (Hanslmayr et al., 2008) and that previous fMRI studies have demonstrated that ACC activation is modulated by previous trial conflict adaptation (Kerns et al., 2004). The absence of a conflict adaptation effect on the $\mathrm{N}_{\text {INC }}$ was unexpected and is inconsistent with the microlevel SOA sensitivity observed in this study for this component, leaving open the need for further research to better determine how reactive control maps onto the processes indexed by the $\mathrm{N}_{\text {INC. }}$.

\section{Proactive Control on a Macroscopic Timescale}

Proactive control has been suggested to take the form of an early attentional selection in which information relevant to current behavioral goals are actively maintained to anticipate needs before cognitively demanding events occur (Braver et al., 2007; Miller \& Cohen, 2001). The manner in which proactive control is exerted in the human brain is a source of active debate (Bugg \& Hutchison, 2013; Grandjean et al., 2012; Bugg et al., 2010) that has particularly revolved around the mapping between stimulus-response conflict (i.e., congruency/ incongruency) and stimulus-response contingencies (responses that are most frequently associated with a given stimulus that can be manipulated for specific items, over blocks of trials, or over entire experiments/lists).

In this study, we probed proactive control through two manipulations. First, to assess proactive control as 
it relates to relative strength of stimulus-response conflict, we assessed congruency effects elicited in the colornaming Stroop task and then contrasted them to those evoked using the identical stimuli but when participants performed the word-naming Reverse Stroop task. In line with widely reported asymmetries in effect sizes because of color and word incongruency (Durgin, 2000; MacLeod, 1991), both the RT and $\mathrm{N}_{\text {INC }}$ interference measures showed dramatic, roughly $50 \%$, reductions in effect sizes for word identification relative to color identification tasks when the proportion of trial types were both 50\%/50\%. This asymmetry has traditionally been interpreted within a framework of automaticity in which reading is seen as a highly learned, rather automatic response that elicits rapid processing, whereas color naming is less practiced and requires more focused attention.

Following from these task asymmetries, we performed a third experiment to assess proactive control as it relates to stimulus-response contingencies. For this purpose, a second version of the word-naming Reverse Stroop task was performed in which the proportion of incongruent trials was reduced from $50 \%$ to $20 \%$. This manipulation was constant across all trials in the task and therefore reflected a type of "list-wise" proportion incongruency. As expected from previous behavioral (Glaser \& Glaser, 1982) and ERP (Tillman \& Wiens, 2011; West \& Alain, 2000) applications of the proportion incongruency manipulation, $\mathrm{RT}$ and $\mathrm{N}_{\mathrm{INC}}$ effect sizes were enhanced in the presence of more infrequent conflict. In fact, with the current stimuli, a reduction of the incongruency percentage to only $1 / 5$ of the trials amounted to an almost complete recovery of both the RT and $\mathrm{N}_{\text {INC }}$ incongruency effects to the levels observed in the more automatic Stroop task with a 50\%/50\% proportion. This nearcomplete recovery, coupled with the similar distribution and latencies of the $\mathrm{N}_{\text {INC }}$ under the different task conditions, suggests that task demands and the proportion of relative incongruency may act to modulate a common conflict mechanism. It should be noted, however, that the list-wise proportion incongruency manipulation used here is not informative about other types of flexible, item-specific mechanisms that are now appreciated to also contribute to proactive control (Blais \& Bunge, 2010).

Given the striking recovery in the amplitude of the $\mathrm{N}_{\text {INC }}$, it is interesting that the LPC component followed a different pattern of effects across these three tasks. Unlike the behavioral and $\mathrm{N}_{\mathrm{INC}}$ effects, the LPC did not differ between the two equal probability tasks, but was substantially enhanced when the proportion of the weaker color interference was reduced in the Reverse Stroop 80/20 task. Because incongruency occurred relatively infrequently under this latter task, it would be expected that less ongoing top-down control would have been in place. Nonetheless, the amplitudes of the LPC roughly doubled, suggesting that it may be signaling greater processing because of the reduced level of ongoing, preparatory, proactive control. As the LPC activ- ity generally followed the RT in time and did not correlate with within-trial behavior effect sizes, this component may reflect a form of late task-related assessment and readjustment, consistent with the observation that this component has been found to correlate with next-trial incongruency RT effect sizes (Larson et al., 2009).

Collectively, the combination of electrophysiological and behavioral results described in this study reveal distinct control mechanisms that unfold over time in response to conflicting stimulus input occurring under different contexts and circumstances. We found that behavioral and ERP effects closely paralleled each other in response to both within-trial manipulations that modulate the need for reactive control, as well as to betweentask manipulations that invoke differing amounts of proactive control. In particular, we observed that across experimental conditions, $\mathrm{N}_{\text {INC }}$ amplitudes closely paralleled RTs indicating that this component is sensitive to the overall level of stimulus conflict. In contrast, LPC amplitudes were largest with infrequent incongruent trials, suggesting a possible readjustment role when proactive control is reduced.

\section{Acknowledgments}

We would like to thank Tobias Egner for his helpful comments on this research. This work was supported by grants from the National Institute of Health (grants R01-MH60415 and R01NS051048) to M. G. W.

Reprint requests should be sent to L. Gregory Appelbaum, 200 Trent Dr., Duke University Hospital South, Durham, NC 27710, or via e-mail: greg@duke.edu.

\section{Note}

1. LPC has been commonly used to refer to the P3a, P3b, and various other slow wave components that are not necessarily evoked in response to stimulus incongruency (e.g., Dien, Spencer, \& Donchin, 2004). Here we use the terminology "late positive component" (LPC) to refer only to the slow-wave portion of the wave evoked directly in response to Stroop conflict.

\section{REFERENCES}

Appelbaum, L. G., Boehler, C. N., Won, R., Davis, L., \& Woldorff, M. G. (2012). Strategic orientation of attention reduces temporally predictable stimulus conflict. Journal of Cognitive Neuroscience, 24, 1834-1848.

Appelbaum, L. G., Meyerhoff, K. L., \& Woldorff, M. G. (2009). Priming and backward influences in the human brain: Processing interactions during the Stroop interference effect. Cerebral Cortex, 19, 2508-2521.

Atkinson, C. M., Drysdale, K. A., \& Fulham, W. R. (2003). Event-related potentials to Stroop and reverse Stroop stimuli. International Journal of Psychophysiology, 47, $1-21$.

Badzakova-Trajkov, G., Barnett, K. J., Waldie, K. E., \& Kirk, I. J. (2009). An ERP investigation of the Stroop task: The role of the cingulate in attentional allocation and conflict resolution. Brain Research, 1253, 139-148. 
Bailey, K., West, R., \& Anderson, C. A. (2010). A negative association between video game experience and proactive cognitive control. Psychophysiology, 47, 34-42.

Blais, C., \& Bunge, S. (2010). Behavioral and neural evidence for item-specific performance monitoring. Journal of Cognitive Neuroscience, 22, 2758-2767.

Botvinick, M. M., Braver, T. S., Barch, D. M., Carter, C. S., \& Cohen, J. D. (2001). Conflict monitoring and cognitive control. Psychological Review, 108, 624-652.

Braver, T. S. (2012). The variable nature of cognitive control: A dual mechanisms framework. Trends in Cognitive Sciences, 16, 106-113.

Braver, T. S., Gray, J. R., \& Burgess, G. (2007). Explaining the many varieties of working memory variation: Dual mechanisms of cognitive control. In A. R. A. Conway, C. Jarrold, M. J. Kane, \& J. Towse (Eds.), Variation in working memory (pp. 76-106). Oxford: Oxford Univ Press.

Braver, T. S., Paxton, J. L., Locke, H. S., \& Barch, D. M. (2009). Flexible neural mechanisms of cognitive control within human prefrontal cortex. Proceedings of the National Academy of Sciences, U.S.A., 106, 7351-7356.

Bruchmann, M., Herper, K., Konrad, C., Pantev, C., \& Huster, R. J. (2010). Individualized EEG source reconstruction of Stroop interference with masked color words. Neuroimage, 49, 1800-1809.

Bugg, J. M., \& Hutchison, K. A. (2013). Converging evidence for control of color-word Stroop interference at the item level. Journal of Experimental Psychology: Human Perception and Performance, 39, 433-449.

Bugg, J. M., Jacoby, L. L., \& Chanani, S. (2010). Why it is too early to lose control in accounts of item-specific proportion congruency effects. Journal of Experimental Psychology: Human Perception and Performance, 37, 844-859.

Carter, C. S., Macdonald, A. M., Botvinick, M., Ross, L. L., Stenger, V. A., Noll, D., et al. (2000). Parsing executive processes: Strategic vs. evaluative functions of the anterior cingulate cortex. Proceedings of the National Academy of Sciences, U.S.A., 97, 1944-1948.

Carter, C. S., \& van Veen, V. (2007). Anterior cingulate cortex and conflict detection: An update of theory and data. Cognitive, Affective \& Behavioral Neuroscience, 7, 367-379.

Chen, S., \& Melara, R. D. (2009). Sequential effects in the Simon task: Conflict adaptation or feature integration? Brain Research, 1297, 89-100.

Coderre, E., Conklin, K., \& van Heuven, W. J. (2011). Electrophysiological measures of conflict detection and resolution in the Stroop task. Brain Research, 1413, $51-59$.

Crump, M. J., Gong, Z., \& Milliken, B. (2006). The contextspecific proportion congruent Stroop effect: Location as a contextual cue. Psychonomic Bulletin \& Review, 13 316-321.

Dien, J., Spencer, K. M., \& Donchin, E. (2004). Parsing the late positive complex: Mental chronometry and the ERP components that inhabit the neighborhood of the $\mathrm{P} 300$. Psychophysiology, 41, 665-678.

Donohue, S. E., Appelbaum, L. G., Park, C. J., Roberts, K. C., \& Woldorff, M. G. (2013). Cross-modal stimulus conflict: The behavioral effects of stimulus input timing in a visual-auditory Stroop task. PloS One, 8, e62802.

Donohue, S. E., Liotti, M., Perez, R., III, \& Woldorff, M. G. (2012). Is conflict monitoring supramodal? Spatio-temporal dynamics of cognitive control processes in an auditory Stroop task. Cognitive, Affective \& Behavioral Neuroscience, 12, $1-15$.

Durgin, F. H. (2000). The reverse Stroop effect. Psychonomic Bulletin \& Review, 7, 121-125.
Egner, T. (2007). Congruency sequence effects and cognitive control. Cognitive, Affective \& Behavioral Neuroscience, 7, 380-390.

Egner, T. (2008). Multiple conflict-driven control mechanisms in the human brain. Trends in Cognitive Sciences, 12, 374-380.

Funes, M. J., Lupianez, J., \& Humphreys, G. (2010). Sustained vs. transient cognitive control: Evidence of a behavioral dissociation. Cognition, 114, 338-347.

Glaser, M. O., \& Glaser, W. R. (1982). Time course analysis of the Stroop phenomenon. Journal of Experimental Psychology: Human Perception and Performance, 8, 875-894.

Grandjean, J., D’Ostilio, K., Phillips, C., Balteau, E., Degueldre, C., Luxen, A., et al. (2012). Modulation of brain activity during a Stroop inhibitory task by the kind of cognitive control required. PloS One, 7, e41513.

Greenblatt, R. E., \& Pflieger, M. E. (2004). Randomizationbased hypothesis testing from event-related data. Brain Topography, 16, 225-232.

Hanslmayr, S., Pastotter, B., Bauml, K. H., Gruber, S., Wimber, M., \& Klimesch, W. (2008). The electrophysiological dynamics of interference during the Stroop task. Journal of Cognitive Neuroscience, 20, 215-225.

Jacoby, L. L., Kelley, C. M., \& McElree, B. D. (1999). The role of cognitive control: Early selection versus late correction. In S. Chaiken \& Y. Trope (Eds.), Dual-process theories in social psychology (pp. 383-400). New York: Guilford Press.

Kerns, J. G., Cohen, J. D., MacDonald, A. W., III, Cho, R. Y., Stenger, V. A., \& Carter, C. S. (2004). Anterior cingulate conflict monitoring and adjustments in control. Science, 303, 1023-1026.

King, J. A., Korb, F. M., \& Egner, T. (2012). Priming of control: Implicit contextual cuing of top-down attentional set. The Journal of Neuroscience, 32, 8192-8200.

Larson, M. J., Kaufman, D. A., \& Perlstein, W. M. (2009). Neural time course of conflict adaptation effects on the Stroop task. Neuropsychologia, 47, 663-670.

Liotti, M., Woldorff, M. G., Perez, R., \& Mayberg, H. S. (2000). An ERP study of the temporal course of the Stroop color-word interference effect. Neuropsychologia, 38, 701-711.

Luck, S. J. (2005). An introduction to the event-related potential technique. Cambridge, MA: MIT Press.

MacLeod, C. M. (1991). Half a century of research on the Stroop effect: An integrative review. Psychological Bulletin, 109, 163-203.

Mansouri, F. A., Tanaka, K., \& Buckley, M. J. (2009). Conflictinduced behavioural adjustment: A clue to the executive functions of the prefrontal cortex. Nature Reviews Neuroscience, 10, 141-152.

Markela-Lerenc, J., Ille, N., Kaiser, S., Fiedler, P., Mundt, C., \& Weisbrod, M. (2004). Prefrontal-cingulate activation during executive control: Which comes first? Brain Research, Cognitive Brain Research, 18, 278-287.

Miller, E. K., \& Cohen, J. D. (2001). An integrative theory of prefrontal cortex function. Annual Review of Neuroscience, 24, 167-202.

Nichols, T. E., \& Holmes, A. P. (2002). Nonparametric permutation tests for functional neuroimaging: A primer with examples. Human Brain Mapping, 15, 1-25.

Purmann, S., Badde, S., \& Wendt, M. (2009). Adjustments to recent and frequent conflict reflect two distinguishable mechanisms. Psychonomic Bulletin E Review, 16, 350-355.

Ratcliff, R. (1978). A theory of mental retrieval. Psychological Review, 85, 59-108.

Ridderinkhof, K. R. (2002). Micro- and macro-adjustments of task set: Activation and suppression in conflict tasks. Psychological Research, 66, 312-323.

Stroop, J. R. (1935). Studies of interference in serial verbal reactions. Journal of Experimental Psychology, 18, 643-662. 
Sturmer, B., Leuthold, H., Soetens, E., Schroter, H., \& Sommer, W. (2002). Control over location-based response activation in the Simon task: Behavioral and electrophysiological evidence. Journal of Experimental Psychology: Human Perception and Performance, 28, 1345-1363.

Sugg, M. J., \& McDonald, J. E. (1994). Time course of inhibition in color-response and word-response versions of the Stroop task. Journal of Experimental Psychology: Human Perception and Performance, 20, 647-675.

Talsma, D., \& Woldorff, M. G. (2005). Methods for the estimation and removal of artifacts and overlap in ERP waveforms. In T. Handy (Ed.), Event-related potentials: A methods handbook (pp. 115-148). Cambridge, MA: MIT Press.

Tillman, C. M., \& Wiens, S. (2011). Behavioral and ERP indices of response conflict in Stroop and flanker tasks.

Psychophysiology, 48, 1405-1411.
West, R. (2003). Neural correlates of cognitive control and conflict detection in the Stroop and digit-location tasks. Neuropsychologia, 41, 1122-1135.

West, R., \& Alain, C. (1999). Event-related neural activity associated with the Stroop task. Brain Research, Cognitive Brain Research, 8, 157-164.

West, R., \& Alain, C. (2000). Effects of task context and fluctuations of attention on neural activity supporting performance of the Stroop task. Brain Research, 873, 102-111.

West, R., Jakubek, K., Wymbs, N., Perry, M., \& Moore, K. (2005). Neural correlates of conflict processing. Experimental Brain Research, 167, 38-48.

Woldorff, M. G., Liotti, M., Seabolt, M., Busse, L., Lancaster, J. L., \& Fox, P. T. (2002). The temporal dynamics of the effects in occipital cortex of visual-spatial selective attention. Brain Research, Cognitive Brain Research, 15, 1-15. 